\title{
Multifrequency-Based Range Estimation of RFID Tags
}

\author{
Xin Li, Member, IEEE, Yimin Zhang, Senior Member, IEEE, and Moeness G. Amin, Fellow, IEEE
}

\begin{abstract}
Radio frequency identification (RFID) is a rapidly developing wireless communication technology for electronically identifying, locating, and tracking products, assets, and personnel. RFID has become one of the primarily means to construct a real-time locating system (RTLS) that tracks and identifies the location of objects in real time using simple, inexpensive tags attached to or embedded in objects and readers that receive the wireless signals from these tags to determine their locations. Most RFID tag localization techniques heavily rely on precise estimation of the range between a reader and the tags. Traditionally, range information is obtained from the received signal strength indication (RSSI). Such approach is inaccurate, particularly in a complicated propagation environment. Recent development on phase difference of arrival (PDOA) allows coherent signal processing for improved range estimation performance. Exploiting multiple frequencies may further improve the range estimation performance. In this paper, we focus on multifrequency-based techniques to achieve several important advantages in the range estimation of passive or semi-passive RFID tags. The use of well designed multiple frequencies allows effective phase upwrapping and elimination of the range ambiguity problem which may be encountered in PDOA methods. In a complicated propagation environment, the mutifrequency-based techniques provide frequency diversity for robust range estimation when signals are highly faded at some frequencies. These advantages not only yield improved range estimation accuracy of RFID tags in various applications, but also enable robust range estimation in challenging scenarios.
\end{abstract}

Index Terms - Real-time location system (RTLS), RFID, phase difference of arrival, multifrequency system, range estimation, Chinese remainder theorem.

\section{INTRODUCTION}

Radio frequency identification (RFID) is a rapidly developing wireless communication technology thanks to its capability of electronically identifying, locating, and tracking products, assets, animals, vehicles, and personnel, driven by security, safety, cost and other factors [1], [2], [3]. RFID has become one of the primarily means to construct a real-time locating system (RTLS) that tracks and identifies the location of objects in real time using simple, inexpensive tags attached to or embedded in objects and readers that receive the wireless signals from these tags to determine their locations [4], [5].

Localization of an object can be achieved in different ways [6]. One of commonly used approaches is trilateration that determines the tag position by fusing range information of an RFID tag estimated at three or more readers separated by a distance, and the tag position can be solved through the trilateration technique. Alternatively, by using the array

The authors are with the Center for Advanced Communications, Villanova University, Villanova, PA 19085, USA (e-mails: \{xin.l, yimin.zhang, moeness.amin\}@villanova.edu). processing technology at RFID readers for the estimation of direction-of-arrival (DOA), an RFID tag can also be localized through hybrid techniques that combine the range and DOA information. In this case, it is possible to locate an RFID tag with a single reader that provides both range and DOA information. In the above techniques, tag localization heavily relies on precise range estimation from a reader. A traditional approach for the estimation of range information is based on the received signal strength indication (RSSI). Such approach, however, is inaccurate, particularly in a complicated propagation environment. Recent development on phase difference of arrival (PDOA) allows coherent signal processing for improved range estimation performance [7]. PDOA based approaches share the same concept as the dual-frequency techniques for range estimation being applied in radar systems [8], [9], [10] where signals with two basic frequencies are used, and the phase difference observed at the two frequencies is used to estimate the range of the reflecting objects. Exploiting multiple frequencies may further improve the range estimation performance. For example, averaging the range estimates over multiple frequency pairs reduces the effect of noise [7].

Passive RFID tags have no internal power supply and, therefore, can be manufactured with a very low price and have an unlimited life span. The majority of RFID tags in existence are of the passive variety. Semi-passive RFID tags are very similar to passive tags except for the addition of a small battery, which allows the tag IC to be constantly powered and removes the need for the antenna to be designed to collect power from the incoming signal. Antennas can therefore be optimized for the backscattering signal. As a result, semi-passive RFID tags can provide much longer read range compared to passive RFID tags. PDOA-based range estimation approaches of passive or semi-passive RFID tags is made possible primarily because, during the uplink period, the tags are powered by the carrier signals transmitted from the reader and backscatter the carrier signal to the reader [11], [12], [13]. During this period, a tag signals binary amplitude shift keying (ASK) codes by changing the impedance of the antenna so as to alter the reflection efficiency. This fact provides the convenience for the waveform design at the reader for our purpose of range estimation. It is emphasized that, while the IC-based passive and semi-passive tags require certain time to respond to the commands from a reader, the phase difference information used in the PDOA-based range estimation technique is not affected by such a delay.

In this paper, we address some key features of multifrequency-based techniques in the range estimation of passive or semi-passive RFID tags. The use of well designed 
multiple frequencies allows effective phase unwrapping and elimination of the range ambiguity problem encountered in PDOA methods. Phase wrapping is a critical issue in range estimation when the maximum possible range is relatively large. A dual-frequency RFID system, or multifrequency RFID system with equal frequency spacing, yields an maximum unambiguous range which is inversely proportional to the separation between two adjacent frequencies [8], [9], [10]. Such unambiguous range may not be enough, depending on RFID systems, if a certain frequency separation is required to have enough frequency separation for various reasons including reduction of phase noise effect. Note that, the maximum read ranges of passive and semi-passive RFID tags are expected to extend in the future with the advancement of technology. Modulation and coding schemes may be modified to accommodate the needs for long-range object localization and tracking. Toward this end, phase unwrapping using well selected unequally spaced frequencies can satisfy both unambiguous range as well as phase noise reduction [14], [15]. On the other hand, in a complicated propagation environment, the mutifrequencybased techniques provide frequency diversity for robust range estimation when signals are highly faded at some frequencies. The combination of different frequency pairs allows multiple sets of range estimates, which can be fused with appropriate weights, rather than previously proposed equalweight averaging, to further reduce the range estimation errors. These developments not only yield improved range estimation accuracy of RFID tags in various applications, but also enable robust range estimation in challenging scenarios.

This paper is organized as follows. Section II introduces the PDOA-based range estimation techniques exploiting dualfrequency signaling, which serves as the foundation of our discussion for multifrequency-based range estimation approaches. In this section, the concept of range estimation using dualfrequency signaling is first reviewed, and the modulation and decoding of RFID tag signals are then considered. This section also addresses the sensitivity of range estimation performance to noise. Section III considers the multifrequency-based range estimation. Our discussions focus on two important issues, i.e., the selection of carrier frequencies, and the weighted fusion of range estimates obtained from different frequency pairs. Appropriate carrier frequency selection through the use of Chinese remainder theorem (CRT) is introduced and the design approach that accounts for the minimum range distance requirement is provided. A number of methods that fuse the range estimates from multiple frequency pairs are formulated. Section IV provides numerical and simulation results to validate and demonstrate the offerings of the proposed methods. Finally, we provide some remarks and conclude this paper in Section V.

\section{PDOA-BASEd RANGe Estimation Exploiting DUAL-FREQUENCY SIGNALING}

\section{A. Range Estimation Based on Dual-Frequency Signaling}

Consider that an RFID reader transmits a dual-frequency continuous-wave (CW) using frequencies $f_{1}$ and $f_{2}$ to provide power for the RFID tag to send uplink signals. Without

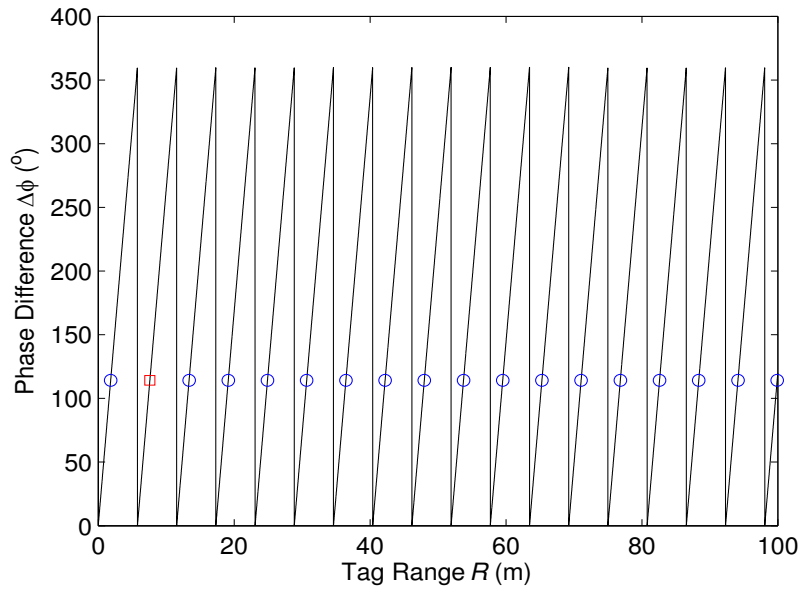

Fig. 1. Phase difference versus the tag range. Both ambiguous tag range values (circle) and actual tag range value (square) are illustrated ( $\Delta f_{12}=26$ $\mathrm{MHz}, R=7.6 \mathrm{~m})$.

considering the modulation performed at the RFID tag and the receiver noise (these effects will be considered later), the uplink signal at frequency $f_{i}, i=1,2$, can be expressed as,

$$
y_{i}(t)=\rho_{i} \exp \left(-j \phi_{i}\right) s(t), \quad i=1,2,
$$

where $s(t)$ is the dual-frequency waveform transmitted from the reader, $\rho_{i}$ and $\phi_{i}$ are, respectively, the range-dependent amplitude and the phase of the return corresponding to the $i$-th frequency of operation. It is assumed that $\Delta f_{12}=f_{2}-f_{1}>0$. If $R$ is the range of the RFID tag, then

$$
\phi_{i}=\frac{4 \pi f_{i} R}{c},
$$

where $c$ is the velocity of electromagnetic wave propagation. Therefore, range $R$ can be estimated from the phase difference observed at the return signal corresponding to the two frequencies, i.e.,

$$
\hat{R}=\frac{c \Delta \phi_{12}}{4 \pi \Delta f_{12}},
$$

where $\Delta \phi_{12}=\phi_{2}-\phi_{1}$ is the observed phase difference.

If the range of an RFID tag is larger than

$$
R_{\max }=\frac{c}{2 \Delta f_{12}},
$$

which is referred to as the maximum unambiguous range, the phase difference between the two carrier frequencies exceeds $2 \pi$. Because phase observations are wrapped within the $[0,2 \pi)$ range, it yields range ambiguity problem. That is, the true phase difference can be expressed as

$$
\Phi_{12}=\Delta \phi_{12}+2 m \pi,
$$

where $m \geq 0$ is an unknown integer. Accordingly, the range estimate is subject to range ambiguity [8], [9], [10], i.e.,

$$
\hat{R}=\frac{c \Delta \phi_{12}}{4 \pi \Delta f_{12}}+\frac{c m}{2 \Delta f_{12}} .
$$

The second term in the above equation induces ambiguity in range. For the same phase difference, the tag range estimates can assume infinite values separated by the maximum 


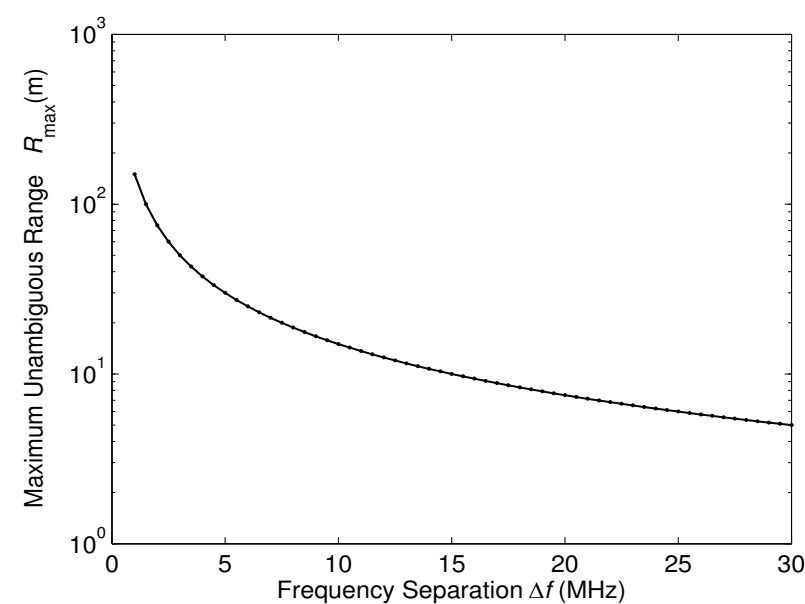

Fig. 2. Maximum unambiguous range versus frequency separation.

unambiguous range $R_{\max }$. An example of the phase difference versus the tag range is plotted in Fig. 1, where the frequency separation is $\Delta f_{12}=26 \mathrm{MHz}$ and the actual tag range is $R=7.6 \mathrm{~m}$. From eq. (4), the maximum unambiguous range in this case is obtained as $R_{\max }=5.77 \mathrm{~m}$. For clarity, the actual tag range $(R)$ at $7.6 \mathrm{~m}$ is marked by a square and the estimated ranges $(\hat{R})$ at repetitive positions $1.83,13.37,19.14, \cdots$ separated by $R_{\max }=5.77 \mathrm{~m}$ are marked by circles. Therefore, the range ambiguity is likely to be a problem when the maximum RFID read range is large.

It is clear from eq. (4) that, as far as the maximum unambiguous range $R_{\max }$ is concerned, it depends on the difference $\Delta f_{12}$ between the two frequencies whereas their absolute values are not relevant. The values of the maximum unambiguous range at some frequency differences of interest are plotted in Figure 2. It shows that a large frequency difference corresponds to a small value of $R_{\max }$, e.g., $R_{\max }=150 \mathrm{~m}$ for $\Delta f_{12}=1 \mathrm{MHz}$, and $R_{\max }=5 \mathrm{~m}$ for $\Delta f_{12}=30 \mathrm{MHz}$. Thus, to obtain a large maximum unambiguous range value, a small frequency separation is required. Because an RFID system has a finite tag range, adequate frequency separation can be chosen to accommodate unambiguous range estimation. However, as discussed later, decreasing the frequency separation amplifies the sensitivity of the phase difference to noise and thus yields degraded range estimation accuracy. To reduce the sensitivity of the phase difference to noise, therefore, it is necessary to select an adequate value of frequency separation. As a result, the selection of frequency separation should satisfy the requirements for a large maximum unambiguous range and low sensitivity of the phase difference to noise.

\section{B. Signal Modulation and Decoding}

A typical return signal reflected at an RFID tag and received at the RFID receiver is the superposition of carrier waveforms transmitted from the reader (due to circulator leakage and environmental scattering) and the modulated backscattering signals. It is further contaminated by scatterers from the environment. Thus, the received RF signal can be expressed as

$$
\begin{aligned}
y_{c}(t) & =\left[\beta_{1}+\gamma_{1}(t)\right] u_{1}(t)+\left[\beta_{2}+\gamma_{2}(t)\right] u_{2}(t) \\
& +\rho_{1} s(t) u_{1}(t) \exp \left(-j \frac{4 \pi f_{1} R}{c}\right) \\
& +\rho_{2} s(t) u_{2}(t) \exp \left(-j \frac{4 \pi f_{2} R}{c}\right)+n(t),
\end{aligned}
$$

where $u_{i}(t)=\exp \left(j 2 \pi f_{i} t\right)$ is the carrier waveform transmitted from the reader at frequency $f_{i}$, where $i=1,2, \beta_{i}$ is a complex scalar representing the strength at the respective carrier frequency, and $\gamma_{i}(t)$ represents the slowly time-varying coefficient of the environment scattering of the forward signal. $s(t) \in[-1,1]$ is the uplink modulating signal whose waveform depends on the protocols used in the RFID system but is common for both frequencies. In addition, $n(t)$ is the additive noise which is typically considered as a bandpass Gaussian random process.

Using coherent correlator to demodulate, the output corresponding to frequency $f_{i}$ is expressed as

$$
\begin{aligned}
z_{i}^{\prime}(t) & =\frac{1}{T} \int_{0}^{T} y_{c}(t) u_{i}^{*}(t) d t \\
& =\beta_{i}+\gamma_{i}(t)+\rho_{i} s(t) \exp \left(-j \frac{4 \pi f_{i} R}{c}\right)+n_{i}^{\prime}(t)
\end{aligned}
$$

where ${ }^{*}$ denotes complex conjugation, $T$ is the symbol period, and

$$
n_{i}^{\prime}(t)=\frac{1}{T} \int_{0}^{T} n(t) u_{i}^{*}(t) d t,
$$

which follows complex Gaussian distribution with zero mean. The first two terms in eq. (8), representing the carrier waveform components due to leakage and environmental scattering, usually have a high power but occupy only a very narrow frequency band around the direct current (DC). Therefore, they can be eliminated from the received signal through proper filtering.

While the detection of $s(t)$ is not necessary for the purpose of range estimation because the waveforms at both frequencies are equally affected by the modulation, we consider that $s(t)$ is properly decoded for processing convenience. Because the phase term $\exp \left(-j 4 \pi f_{i} R / c\right)$ is static or quasi-static over time, which is the case even for an RFID tag embedded to a moving object [16], tag signal $s(t)$ can be obtained from either frequency, or the detected results at the two frequencies can be fused to improve the detection performance. Therefore, the decoded waveform, with the phase term maintained for range estimation, becomes

$$
z_{i}(t)=\rho_{i} \exp \left(-j \frac{4 \pi f_{i} R}{c}\right)+n_{i}(t),
$$

where

$$
n_{i}(t)=n_{i}^{\prime}(t) s(t)=r_{i}(t) \exp \left[j \psi_{i}(t)\right],
$$

with $r_{i}(t)$ denoting the envelop and $\psi_{i}(t)$ the phase of the demodulated noise. Note that $n_{i}(t)$ remains to follow the complex Gaussian distribution with zero mean and the same variance as that of $n_{i}^{\prime}(t)$, whereas the resulting $r_{i}(t)$ follows a Rayleigh distribution, and $\psi_{i}(t)$ follows a uniform distribution within $[0,2 \pi)$. 


\section{Analysis of Sensitivity to Noise Effect}

From the above discussion, the estimation of phase $\phi_{i}$ is obtained from $z_{i}(t)$ as [17]

$$
\begin{aligned}
\hat{\phi}_{i}(t) & =\phi_{i}-\tan ^{-1}\left\{\frac{r_{i}(t) \sin \left[\psi_{i}(t)-\phi_{i}\right]}{\rho_{i}+r_{i}(t) \cos \left[\psi_{i}(t)-\phi_{i}\right]}\right\} \\
& \simeq \phi_{i}-\tan ^{-1}\left\{\frac{r_{i}(t)}{\rho_{i}} \sin \left[\psi_{i}(t)-\phi_{i}\right]\right\} \\
& \simeq \phi_{i}-\frac{r_{i}(t)}{\rho_{i}} \sin \left[\psi_{i}(t)-\phi_{i}\right] \\
& =\phi_{i}-\frac{r_{i}(t)}{\rho_{i}} \sin \left[\psi_{i}^{\prime}(t)\right],
\end{aligned}
$$

where both approximations hold when the signal-to-noise ratio is moderate or high, and $\psi_{i}^{\prime}(t)=\psi_{i}(t)-\phi_{i}$ follows a uniform distribution within $[0,2 \pi)$.

Therefore, the range estimate can be obtained as

$$
\begin{aligned}
\hat{R} & =\frac{c\left(\hat{\phi}_{2}-\hat{\phi}_{1}\right)}{4 \pi \Delta f_{12}}+\frac{c m}{2 \Delta f_{12}} \\
& =\frac{c \Delta \phi_{12}}{4 \pi \Delta f_{12}}+\frac{c m}{2 \Delta f_{12}} \\
& +\frac{c}{4 \pi \Delta f_{12}}\left\{\frac{r_{1}(t)}{\rho_{1}} \sin \left[\psi_{1}^{\prime}(t)\right]-\frac{r_{2}(t)}{\rho_{2}} \sin \left[\psi_{2}^{\prime}(t)\right]\right\} \\
& =R+\frac{c m}{2 \Delta f_{12}} \\
& +\frac{c}{4 \pi \Delta f_{12}}\left\{\frac{r_{1}(t)}{\rho_{1}} \sin \left[\psi_{1}^{\prime}(t)\right]-\frac{r_{2}(t)}{\rho_{2}} \sin \left[\psi_{2}^{\prime}(t)\right]\right\} .
\end{aligned}
$$

The last term in this expression represents the effect of noise. In an unfaded propagation environment, $\rho_{i}$ and $r_{i}(t)$ are insensitive to frequency and, thus, evidently, the noise effect is inversely proportional to the frequency separation. That is, a large frequency separation is desirable to reduce the effect of noise.

\section{Multifrequency-BASEd RAnge Estimation}

As we discussed above, the range estimation performance of the PDOA method based on dual-frequency signaling may suffer in different situations. One of the key limitation is the trade-off between the maximum unambiguous range and the sensitivity of range estimation to noise. That is, a large separation between the two frequencies may reduce the sensitivity of range estimation to additive noise, but doing so yields a small unambiguous range that may not be large enough for the RFID system and application of interest. Another problem raises when the signal at either or both of the two carrier frequencies are severely faded, thus yielding unreliable phase, and subsequently, range estimation from the received signals. The use of three or more carrier frequencies can overcome these issues and enhance the accuracy of phase difference through appropriate data fusion over different frequency pairs. Consequently, the estimation of tag range can be improved. Because different frequency pairs are likely to have varying level of range estimation quality, it is desirable to have weighted average of the range estimates rather than simple averaging.

In the following, we consider three key issues, i.e., the range estimation in a multifrequency signaling system, the selection of frequencies, and the weighted fusion of range estimates obtained from different frequency pairs.

\section{A. Range Estimation}

Consider that an RFID reader sends a multifrequency CW signal using frequencies $f_{1}, f_{2}, \cdots, f_{M}$ to supply power for the RFID tag. Without loss of generality, it is assumed that $f_{1}<f_{2}<\cdots<f_{M}$. For the convenience of explanation, we ignore the effect of noise. Similar to the dual-frequency case, the received RF signal can be expressed as

$$
y_{c}(t)=\sum_{i=1}^{M}\left\{\left[\beta_{i}+\gamma_{i}(t)\right] u_{i}(t)+\rho_{i} s(t) u_{i}(t) \exp \left(-j \frac{4 \pi f_{i} R}{c}\right)\right\} .
$$

After demodulation and decoding, the output corresponding to frequency $f_{i}$ is expressed as

$$
z_{i}(t)=\rho_{i} \exp \left(-j \phi_{i}\right), \quad i=1, \cdots, M,
$$

where $\phi_{i}$ is expressed in eq. (2). The tag range $R$ can be estimated from any pair of the $M$ frequencies using the dual-frequency range estimation method as discussed in the previous section. The range estimate obtained from frequency pair $f_{i}$ and $f_{j}$, where $f_{i}<f_{j}, i, j=1,2, \cdots, M$, can be expressed as

$$
\hat{R}_{i j}=\frac{c \Delta \phi_{i j}}{4 \pi \Delta f_{i j}},
$$

where $\Delta \phi_{i j}=\phi_{j}-\phi_{i}$ is the observed phase difference and and $\Delta f_{i j}=f_{j}-f_{i}$ is the frequency difference.

From the $M$ carrier frequencies, a total number of $K=$ $M(M-1) / 2$ pairs can be used to obtain their respective range estimate. These results can be fused to improve the robustness against noise and frequency selective propagation characteristics, and substantially enhance the unambiguous range as we discuss below in the rest of this section.

\section{B. Frequency Selection}

We first consider the equal spacing frequency selection, as suggested in [7]. Assume that the $M$ frequencies are equally separated by $\Delta f=\Delta f_{i, i+1}$ for $i=1, \cdots, M-1$. The total resulting maximum separation between the highest and lowest frequencies is $\Delta F=(M-1) \Delta f$, which is bounded by the maximum available frequency bandwidth and frequencyhopping requirement regulated by Federal Communications Commission (FCC). The maximum unambiguous range is determined by $\Delta f$ as

$$
R_{\text {max }, \text { equal }}=\frac{c}{2 \Delta f}=\frac{(M-1) c}{2 \Delta F} .
$$

In the following, we develop frequency selection based on unequal frequency spacing to improve the noise sensitivity yet the maximum frequency requirement is met. The proposed technique is based on the Chinese Remainder Theorem (CRT) [18]. For the $k$-th frequency pair consisting of adjacent frequencies, i.e., $\Delta f_{k}=f_{k+1}-f_{k}, k=1, \cdots, M-1$, a wrapped phase difference is observed as $\Delta \phi_{k}=\phi_{k+1}-\phi_{k}$. Then, the tag range is related to the phase observation by

$$
R=\frac{c \Delta \phi_{k}}{4 \pi \Delta f_{k}}+\frac{c m_{k}}{2 \Delta f_{k}}=\frac{\nu_{k} \Delta \phi_{k}}{4 \pi}+\frac{m_{k} \nu_{k}}{2},
$$


where $\nu_{k}=c / \Delta f_{k}$. If we choose $\nu_{k}$ as a multiple of a constant $\Delta_{d}$, which represents a range bin and its value is to be determined by the range resolution, then we can express $\nu_{k}$ as $\nu_{k}=\xi_{k} \Delta_{d}$, where $\xi_{k}$ is a nonnegative integer. As such, the range estimation problem described in eq. (18) can be written as

$$
\Delta \phi_{k}=\frac{2 \pi}{\xi_{k}}\left(\frac{2 R}{\Delta_{d}}-m_{k} \xi_{k}\right) .
$$

The maximum unambiguous range is

$$
R_{\max }=\frac{\Delta_{d}}{2} \cdot \operatorname{LCD}\left(\xi_{1}, \cdots, \xi_{M-1}\right)
$$

where $\operatorname{LCD}(\cdot)$ denotes the least common denominator operator. In addition, the minimum distance between two adjacent ambiguous tag range estimates is given by

$$
D_{\min }=\frac{\Delta_{d}}{2} \cdot \operatorname{GCD}\left(\xi_{1}, \cdots, \xi_{M-1}\right),
$$

where $\mathrm{GCD}(\cdot)$ denotes the greatest common divisor operator.

The maximum separation between the highest and lowest frequencies is obtained as

$$
\Delta F=\sum_{k=1}^{M-1} \Delta f_{k}=\frac{c}{\Delta_{d}}\left(\frac{1}{\xi_{1}}+\cdots+\frac{1}{\xi_{M-1}}\right) .
$$

The maximum unambiguous range obtained from the $k$-th pair is expressed as

$$
R_{\max , k}=\frac{c}{2 \Delta f_{k}}=\frac{\nu_{k}}{2}=\frac{\xi_{k} \Delta_{d}}{2} .
$$

The CRT is widely used to achieve effective phase unwrapping. The CRT is one of the oldest theorems in number theory and is a result about congruences in number theory and its generalizations in abstract algebra. The CRT is stated as:

Chinese Remainder Theorem: If $m_{1}, m_{2}, \cdots, m_{M}$ are coprime positive integers, then for any integers $a_{1}, a_{2}, \cdots, a_{M}$, the set of congruences

$$
\left\{\begin{array}{l}
a_{1}=x \bmod m_{1} \\
a_{2}=x \bmod m_{2} \\
\cdots \quad \cdots \\
a_{M}=x \bmod m_{M}
\end{array}\right.
$$

has a unique solution $x$ modulo $m_{1} m_{2} \cdots m_{M}$.

In our application, the phase unwrapping and thus the range estimation described in eq. (19) can further be formulated as the following CRT problem

$$
\frac{\xi_{k} \Delta \phi_{k}}{2 \pi}=\frac{2 R}{\Delta_{d}} \bmod \xi_{k}, \quad k=1, \cdots, M-1,
$$

where $\xi_{k}$ 's are chosen to be coprime. In this case, the maximum unambiguous range given in eq. (20) and the minimum distance between two adjacent ambiguous range estimates given in eq. (21) are, respectively, reduced to

$$
R_{\max }=\frac{\Delta_{d}}{2} \cdot \prod_{k=1}^{M-1} \xi_{k}
$$

and

$$
D_{\min }=\frac{\Delta_{d}}{2}
$$

From the CRT, it is known that, any tag range $R$ that satisfies $R<R_{\max }$ can be uniquely determined.

Due to the use of unequally spaced frequencies in the multifrequency RFID system, as expressed in eqs. (20) and (26), we can achieve significant improvement of the maximum unambiguous range whereas sufficient minimum distance between two adjacent ambiguous tag range estimates is maintained. As depicted in eq. (17), the improvement over that obtained from a multifrequency RFID system exploiting equally spaced frequencies is evident. For example, when $M=3, f_{1}=902$ $\mathrm{MHz}$ and $f_{3}=928 \mathrm{MHz}$, the selection of $f_{2}$ is considered, where $f_{1}<f_{2}<f_{3}$. Based on the proposed selection scheme, we can select $f_{2}=908$ or $922 \mathrm{MHz}$, which yields the two frequency separations of 20 and $6 \mathrm{MHz}$, respectively, and results in a large maximum unambiguous range of $R_{\max }=75$ $\mathrm{m}$ and a sufficiently large minimum distance of $D_{\min }=2.5$ $\mathrm{m}$ between two adjacent ambiguous tag range estimates. In contrast, for the equal spacing frequency selection described in [7], i.e., $f_{2}=915 \mathrm{MHz}$, we obtain $\Delta f=13 \mathrm{MHz}$ and the corresponding maximum unambiguous range is obtained from eq. (17) as $R_{\text {max, equal }}=11.53 \mathrm{~m}$. Fig. 3 depicts two examples of tag range estimation in a multifrequency RFID system with the illustration of various relevant parameters for the convenience of understanding.

It is noted that, within the available bandwidth, a smaller value of $\Delta_{d}$ provides more possible choices of $\xi_{k}$ to yield a larger maximum unambiguous range. The minimum value of $\Delta_{d}$, however, is determined by the achievable range estimation performance. When $\Delta_{d}$ is too small, we may end up with a wrong range and the range estimation error would be very large. It is also worth mentioning that, because different pairs of frequency separation have varying spacing and thus different estimation errors, we may use different values of $\Delta_{d}$ in the selection of unequal frequencies. Such processing, however, is be discussed in this paper.

\section{Fusion of Range Estimates}

There are a variety ways to fuse the range estimates obtained from the $K=M(M-1) / 2$ possible frequency pairs. Note that in this subsection, we assume that the ambiguous range issue is properly solved, and the phase difference in each pair $\Delta \phi_{i j}$ is unwrapped. The determination of range bins in the presence of noise was discussed, for example, in [14], [15].

For the $k$-th frequency pair $\left(f_{i}, f_{j}\right)$, the unwrapped phase difference $\Delta \Phi_{i j}$ and wrapped one $\Delta \phi_{k}=\Delta \phi_{i j}$ is related by

$$
\Delta \Phi_{i j}=\Delta \phi_{k}+2 m_{k} \pi
$$

The corresponding tag range estimate $\hat{R}_{i j}=\hat{R}_{k}$ is

$$
\hat{R}_{k}=\frac{c \Delta \Phi_{k}}{4 \pi \Delta f_{k}}=\hat{R}_{k 0}+m_{k} R_{\max , k},
$$

where

$$
\hat{R}_{k 0}=\frac{c \Delta \phi_{k}}{4 \pi \Delta f_{k}}=R \bmod R_{\max , k},
$$

is the minimum value of the ambiguous range estimates. Through the fusion operation of the tag range estimates, the final estimate accuracy can be improved. We consider the following five fusion methods (FM's). 


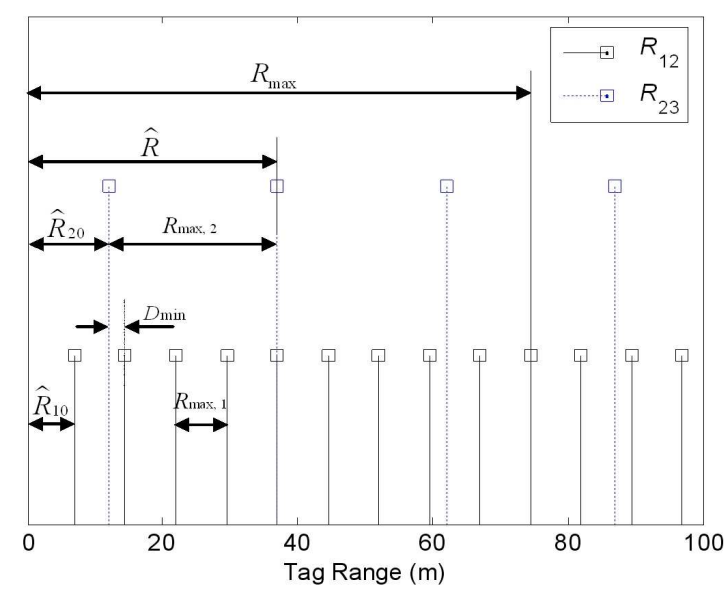

(a) $R=37 \mathrm{~m}$

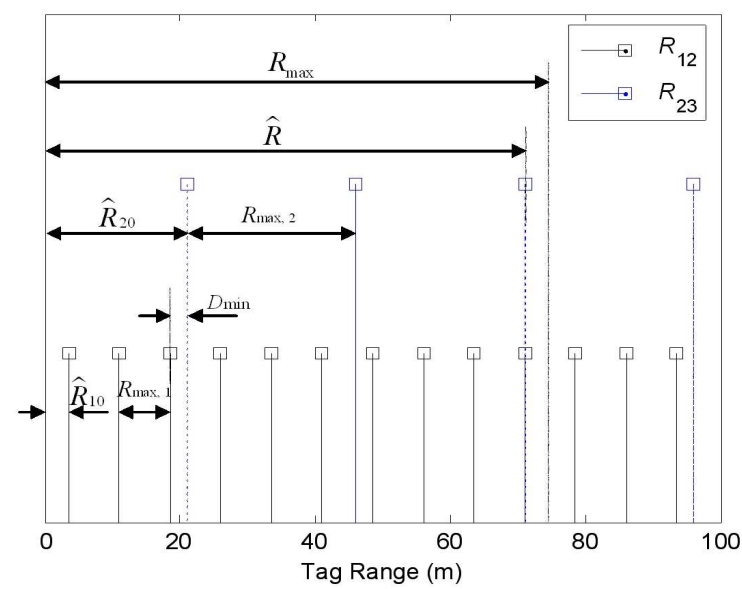

(b) $R=71 \mathrm{~m}$

Fig. 3. Example of tag range estimation in multifrequency RFID system with illustration of parameters $\left(f_{1}=902 \mathrm{MHz}, f_{2}=922 \mathrm{MHz}, f_{3}=928\right.$ $\mathrm{MHz})$.

- FM1: Range-based Averaging

$$
\hat{R}=\frac{1}{K} \sum_{i<j} \hat{R}_{i j}=\frac{c}{4 K \pi} \sum_{i<j} \frac{\Delta \Phi_{i j}}{\Delta f_{i j}} .
$$

- FM2: Phase-based Averaging

$$
\hat{R}=\frac{c \sum_{i<j} \Delta \Phi_{i j}}{4 \pi \sum_{i<j} \Delta f_{i j}} .
$$

- FM3: Range-based Averaging with Weighting

$$
\hat{R}=\sum_{i<j} w_{i j} \hat{R}_{i j}=\frac{c}{4 \pi} \frac{1}{\sum_{i<j} w_{i j}} \sum_{i<j} w_{i j} \frac{\Delta \Phi_{i j}}{\Delta f_{i j}} .
$$

- FM4: Phase-based Averaging with Weighting

$$
\hat{R}=\frac{c \sum_{i<j} w_{i j} \Delta \Phi_{i j}}{4 \pi \sum_{i<j} w_{i j} \Delta f_{i j}} .
$$

- FM5: Selection of Estimate corresponding Largest Frequency Separation

$$
\hat{R}=\hat{R}_{1 M}=\frac{c \Delta \Phi_{1 M}}{4 \pi \Delta f_{1 M}} .
$$

As discussed in Section II-C, the range estimates using the frequency pairs with large separations are insensitive to noise and thereby have a high estimation accuracy. Therefore, the weight of the large separation pair could take a high value in the fusion so as to improve the fusion accuracy. In particular, when

$$
w_{i j}=\frac{\Delta f_{i j}}{\sum_{u<v} \Delta f_{u v}},
$$

the third fusion method (FM3) collapses into the FM2. Additionally, when

$$
w_{i j}=\left\{\begin{array}{ll}
1, & i=1, j=M \\
0, & \text { otherwise }
\end{array},\right.
$$

both FM3 and FM4 reduce into FM5, i.e., only the estimate corresponding the largest frequency separation is considered. In general, the latter four methods outperform the first one, since the joint estimation accuracy of the FM1 depends primarily on the worst accuracy of the $\hat{R}_{i j}$, corresponding the frequency pair with the narrowest frequency separation.

\section{Numerical and Simulation Results}

In this section, we evaluate the performance of tag range estimation based on numerical simulations with frequencies selected within the US UHF band, i.e., 902-928 MHz. The impact of frequency separation on the estimation accuracy of a dual-frequency RFID reader is first illustrated. The CRT-based frequency selection is then demonstrated and the corresponding maximum unambiguous range as well as the minimum distance between two adjacent ambiguous range estimated are depicted. The estimation accuracy under various fusion methods is also examined and compared. In addition, the estimation performance of equally and unequally spaced frequencies are compared.

\section{A. Estimation Accuracy under Various Frequency Separations}

As discussed in Section II-C, the sensitivity of range estimation to noise effect is inversely impacted by the frequency separation. We use the root mean square error (RMSE) of the estimates, described below, as a measure of the accuracy of tag range estimations,

$$
\operatorname{RMSE}(\hat{R})=\sqrt{\frac{1}{N_{t}} \sum_{t=1}^{N_{t}}\left(|R-\hat{R}|^{2}\right)},
$$

where $N_{t}$ is the number of estimation trials used for averaging. Additionally, the signal-to-noise (SNR) is defined as

$$
\mathrm{SNR}=\frac{\left|\rho_{i}\right|^{2}}{\sigma^{2}}
$$

where $\sigma^{2}$ is the variance of the Gaussian noise $n_{i}(t)$ depicted in eq. (11).

In the simulation, each estimate of tag range is averaged across 10 symbols, and $N_{t}=10,000$ independent trials are 


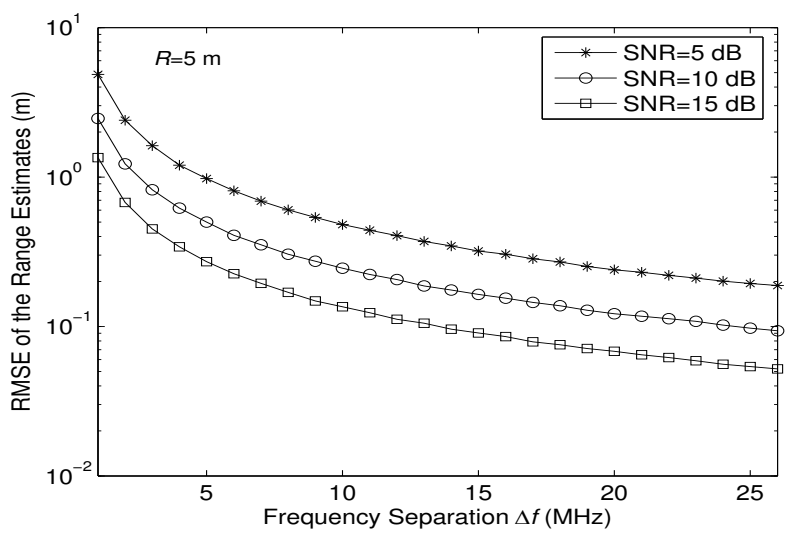

Fig. 4. RMSE of the tag range estimates verse frequency separation.

emulated for the same tag range. For the consideration of the impact of frequency separation, the actual tag range is fixed at $R=5 \mathrm{~m}$, whereas $\Delta f$ varies from $1 \mathrm{MHz}$ to $26 \mathrm{MHz}$ with a 1 $\mathrm{MHz}$ increment. Three different values of SNR, i.e., 5, 10, and $14 \mathrm{~dB}$, are used in the simulations. The RMSE of the tag range estimates versus the frequency separation is illustrated in Fig. 4. As is seen, at the same SNR level, the RMSE of the range estimates monotonically decreases as the frequency separation increases. It is validated that a larger frequency separation provides a higher accuracy of range estimation. In addition, the slope of the curves is not sensitive to different levels of SNR.

\section{B. CRT-based Frequency Selection}

Assume that the number of frequencies is $M=3$ and, for the simplicity of description, the first frequency $f_{1}=902 \mathrm{MHz}$ is fixed. The actual frequency can be changed to accommodate the FCC regulations because, as we discussed earlier, the range estimation is sensitive to the frequency difference but not their absolute frequencies. We still need to select two other frequencies $f_{2}$ and $f_{3}$, where $f_{1}<f_{2}<f_{3}$ and $f_{3}-f_{1} \leq 26$ $\mathrm{MHz}$ is satisfied, so as to provide sufficiently large maximum unambiguous range $R_{\max }$ under a given constraint of the minimum distance of $D_{\min }$ between ambiguous tag range estimates. For the purpose of demonstration, we choose $\Delta_{d}=5$ $\mathrm{m}$ and arbitrarily use $R=16 \mathrm{~m}$ for an example. Then, from eq. (21), one can obtain $D_{\min }=2.5 \mathrm{~m}$. Further, the maximum unambiguous range given in eq. (26) can be calculated for any given $\xi_{k}$ 's. The maximum unambiguous range described in eq. (23) for each frequency pair and corresponding minimum value of the ambiguous range estimates $\hat{R}_{k 0}$ given in eq. (30) can also be obtained. Following such a procedure and according to the CRT described in the Section III-B, frequencies $f_{2}$ and $f_{3}$ can be found. Table I shows the selected searched results with the constraint that the frequencies take integer values in $\mathrm{MHz}$ unit. More choices are available if such constraint is removed. Due to the symmetry symmetry of $\Delta f_{12}$ and $\Delta f_{23}$, only the half results that satisfy $f_{2}-f_{1}>$ $f_{3}-f_{2}$ are included in the table. It is shown that the well designed pairs can achieve a maximum unambiguous range
TABLE I

FREQUENCY SELECTION BASED ON CRT $\left(R=16 \mathrm{~m}, \Delta_{d}=5 \mathrm{~m}\right)$

\begin{tabular}{|cc|c|c|c|cc|cc|l|}
\hline$\xi_{1}$ & $\xi_{2}$ & $f_{1}$ & $f_{2}$ & $f_{3}$ & $R_{\max , 1}$ & $\hat{R}_{10}$ & $R_{\max , 2}$ & $\hat{R}_{20}$ & $R_{\max }$ \\
\hline 3 & 10 & 902 & 922 & 928 & 7.5 & 1 & 25 & 16 & 75 \\
3 & 20 & 902 & 922 & 925 & 7.5 & 1 & 50 & 16 & 150 \\
4 & 15 & 902 & 917 & 921 & 10 & 6 & 37.5 & 16 & 150 \\
5 & 6 & 902 & 914 & 924 & 12.5 & 3.5 & 15 & 1 & 75 \\
5 & 12 & 902 & 914 & 919 & 12.5 & 3.5 & 30 & 16 & 150 \\
\hline
\end{tabular}

of $R_{\max }=150 \mathrm{~m}$, which is significantly higher than that obtained in the equally spaced frequency selection scheme, where $f_{2}=915 \mathrm{MHz}$ under our assumption and thus the corresponding $R_{\max }=11.53 \mathrm{~m}$.

\section{Comparison of Fusion Methods}

The RMSE performance of the range estimates is compared using various fusion methods presented in Section III-C. In the simulations, each estimate of tag range is averaged across 10 symbols and 10,000 independent trials are simulated at the same SNR level to obtain the RMSE. For the convenience of comparison, we assume $f_{1}=902 \mathrm{MHz}$ and $f_{3}=928 \mathrm{MHz}$. For the equally spaced frequency selection, $f_{2}=915 \mathrm{MHz}$, whereas for the unequal spacing selection, $f_{2}=922 \mathrm{MHz}$, corresponding the first frequency selection scheme illustrated in Table II. The RMSE performance for frequencies with equal and unequal spacings is illustrated in Figs. 5 and 6, respectively. As shown in Fig. 5, the five fusion methods have close RMSE performance, since the relatively large frequency separations make the phase difference insensitive to the noise. However, in the unequal spacing selection, the observed phase difference is slightly sensitive to the noise due to the relatively small frequency separation. Therefore, the use of a proper fusion method becomes more important in this case. It is observed from Fig. 6 that the fusion method FM1 yields the worst performance since, as discussed in the Section III-C, the overall estimation accuracy is compromised by the frequency pair with the narrowest separation. Such accuracy degeneration can be mitigated by using a proper fusion method. As seen in Fig. 6, except for FM1 and FM5 (FM5 has the same performance in both cases because of the same frequency separation of $26 \mathrm{MHz}$ ), the other three fusion methods using unequally spaced frequencies outperform that obtained in their equal spacing counterpart. It is due to the fact that in our proposed unequal spacing selection, the constraint of minimum spatial spacing $D_{\text {min }}$ between ambiguous range estimates provides a predetermined accuracy level.

\section{CONCLUSION AND REMARKS}

In this paper, we have proposed the use of multifrequency RFID system that provide the capability of accurate range estimation of RFID tags over a large read range. Range estimation is important to providing localization and tracking of assets and objects in various applications. This paper focus on the use of unequally spaced frequencies to maximize unambiguous range to uniquely determinable the range of an RFID tag, and examined different techniques to fuse the range estimates obtained from multiple frequency pairs. As a result, 


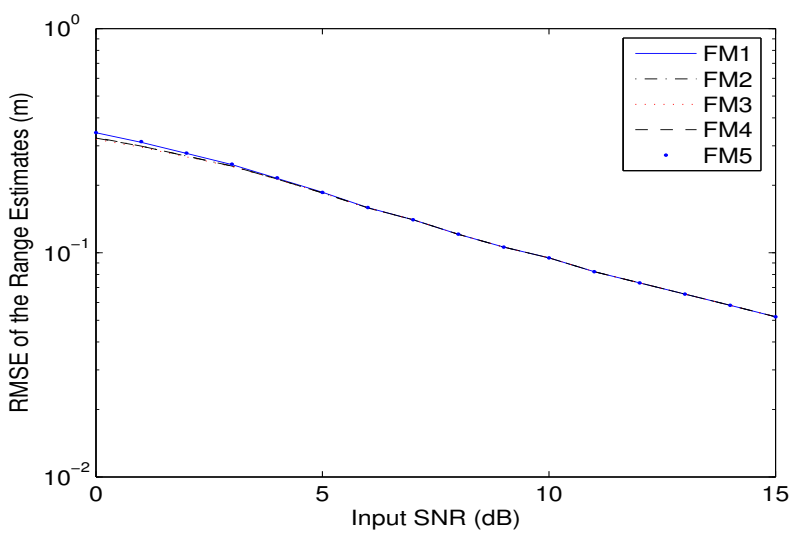

Fig. 5. RMSE of the range estimates for various fusion methods (equally spaced frequencies: $f_{1}=902 \mathrm{MHz}, f_{2}=915 \mathrm{MHz}, f_{3}=928 \mathrm{MHz}$ ).

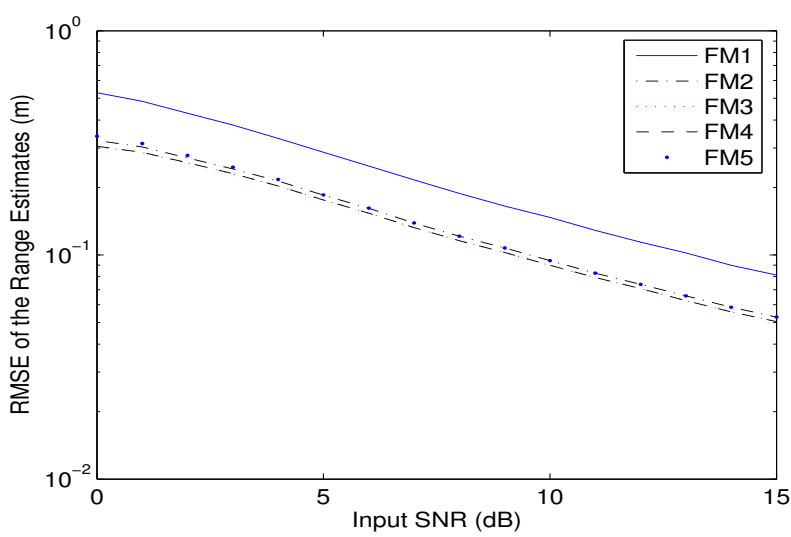

Fig. 6. RMSE of the range estimates for various fusion methods (unequally spaced frequencies: $f_{1}=902 \mathrm{MHz}, f_{2}=922 \mathrm{MHz}, f_{3}=928 \mathrm{MHz}$ ).

satisfactory range estimation performance can be achieved over a large RFID read range.

It is worth mentioning that, while we could not discuss it in this paper due to space limitations, the multifrequency concept can be implemented using frequency-hopping techniques developed in, for example, [19]. By divide a uplink packet into several segments, frequency-hopping techniques allow the transmission of single frequency at each time instants and the carrier frequency remains unchanged over each segment but changes among different segments. Assuming static or quasistatic RFID tags which are valid in typical RFID applications, the phase difference information is obtained by comparing the phase information over different segments which use different frequencies. The use of frequency-hopping techniques may simplify the hardware complexity and yield transmit signals with a constant modulus.

\section{ACKNOWLEDGMENT}

This work is supported in part by the Ben Franklin Technology Partners of Southeastern Pennsylvania (BFTP/SEP).

\section{REFERENCES}

[1] K. Finkenzeller, RFID Handbook, 2nd Ed., New York, NY: Wiley, 2003.

[2] S. A. Ahson and M. Ilyas, RFID Handbook: Applications, Technology, Security, and Privacy, CRC Press, 2008.

[3] D. E. Brown, RFID Implementation, New York, NY: McGraw Hill, 2007.

[4] L. M. Ni, Y. Liu, Y. C. Lau, A. P. Patil, "LANDMARC: Indoor Location Sensing Using Active RFID," 1st IEEE Int. Conf. on Pervasive Computing and Communications (PerCom'03), pp. 407-415, 2003.

[5] D. Hähnel, W. Burgard, D. Fox, K. Fishkin, and M. Philipose, "Mapping and localization with RFID technology," Intel White Paper, IRS-TR-03-014, Dec. 2003. Available at http: //www.intel-research.net/Publications / Seattle/012020041250_211.pdf.

[6] A. H. Sayed, A. Tarighat, and N. Khajehnouri, "Network-based wireless location," IEEE Signal Proc. Mag., vol. 22, no. 4, pp. 24-40, July 2005.

[7] M. Knox and R. Bridglall, "Object localization based security using RFID," US patent 2006/0607307 A1, May 2006.

[8] F. Ahmad, M. Amin, P. Setlur, "Through-the-wall target localization using dual-frequency CW radars," Proc. SPIE, vol. 6201, Orlando, FL, April 2006.

[9] F. Ahmad, M. G. Amin, and P. D. Zemany, "Performance analysis of dual-frequency CW radars for motion detection and ranging in urban sensing applications," Proc. SPIE, vol. 6547, Orlando, FL, April 2007.

[10] Y. Zhang, M. G. Amin, and F. Ahmad, "Time-frequency analysis for the localization of multiple moving targets using dualfrequency radars," IEEE Signal Processing Letters, vol. 15, pp. 777-780, 2008.

[11] D. M. Dobkin, The RF in RFID: Passive UHF RFID in Practice, Burlington, MA: Newnes, 2008.

[12] " $860 \mathrm{MHz}-930 \mathrm{MHz}$ Class I Radio Frequency Identification Tag Radio Frequency \& Logical Communication Interface Specification Candidate Recommendation, Version 1.0.1," Auto-ID Center Technical Report, Nov. 2002. Available at http: / / www . epcglobalinc.org/standards_technology/Secure/ v1.0/UHF-class1.pdf.

[13] EPCglobal, Radio-Frequency Identity Protocols Class-1 Generation-2 UHF RFID, Dec. 2005.

[14] G. Wang, X.-G. Xia, V. C. Chen, and R. L. Fiedler, "Detection, location, and imaging of fast moving targets using multifrequency antenna array SAR,' IEEE Trans. Aerosp. Electron. Syst., vol. 40, no. 1, pp. 345-355, Jan. 2004.

[15] X.-G. Xia and G. Wang, "Phase unwrapping and a robust Chinese remainder theorem," IEEE Signal Processing Letters, vol. 14, no. 4, pp. 247-250, April 2007.

[16] Y. Zhang, M. G. Amin, and S. Kaushik, "Localization and tracking of passive RFID tags based on direction estimation," International Journal of Antennas and Propagation, vol. 2007, Article ID 17426, doi:10.1155/2007/17426, 9 pages, Dec. 2007.

[17] S. Haykin, Communication Systems, 4th Ed., New York, NY: Wiley, 2001.

[18] C. Ding, D. Pei, and A. Salomaa, Chinese Remainder Theorem: Applications in Computing, Coding, Cryptography, World Scientific, 1999.

[19] Y. Zhang, M. G. Amin, and F. Ahmad, "Narrowband frequencyhopping radars for the range estimation of moving and vibrating targets," SPIE Symposium on Defense and Security Symposium, Orlando, FL, March 2008. 